\title{
MICROFABRICATED OUT-OF-PLANE ARRAYS OF INTEGRATED CAPILLARY NANO-ELECTROSPRAY EMITTERS
}

\author{
R. Krpoun and H.R. Shea ${ }^{*}$ \\ Ecole Polytechnique Fédérale de Lausanne (EPFL), Microsystems for Space Technologies Laboratory, \\ Neuchâtel, Switzerland
}

\begin{abstract}
This paper presents the fabrication and operation of an integrated nano-electrospray thruster consisting of an array of microfabricated silicon capillary emitters and microfabricated silicon extractor electrodes. Based on previous work in which we showed operation of single microfabricated capillaries [1], the improved thruster presented here allows simultaneously operation of arrays of emitters. In addition, we control the hydraulic impedance of the capillaries by filling them with silica beads, thus tailoring the flow rate in order to spray either in droplet regime or in ionic regime for two ionic liquids. Operation in both modes is confirmed by mass spectrometry and retarding potential analysis. In ion regime, a specific impulse of $3500 \mathrm{~s}$ is obtained at $1.2 \mathrm{kV}$

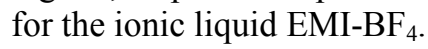

\section{KEYWORDS}

Electrospray, MEMS propulsion, colloid thruster

\section{INTRODUCTION}

Electrospray is a well known technique for sample injection for mass spectrometry [2]. A conductive liquid inside a capillary is placed in front of an annular extraction electrode. A large $(>1 \mathrm{kV})$ potential difference is applied and the liquid is electrostatically attracted to the extractor. As the voltage is increased the liquid tip becomes sharper, eventually snapping into a Taylor cone, and emission of charged droplets or of ion begins once a critical electric field is reached at the tip of the cone.

The recent discovery of an emission regime in which ions instead of droplets are emitted [3] has sparked interest to use electrospray sources for applications such as spacecraft propulsion [4] or for surface micro-machining [5]. Because the mass throughput of electrosprays in ionic mode is very low, one single emitter is insufficient to generate the necessary mass throughput to generate sufficient thrust even for picosatellite missions, hence the need for arrays of emitters.

Pure ion mode has previously only been shown with externally wetted emitters [6] and single, macroscopic capillaries [3]. We present here the microfabrication and testing of arrays of 19 capillaries that can be operated in either pure ion mode or in droplet mode depending on the applied voltage and on the hydraulic impedance of the silicon capillaries.

\section{THRUSTER LAYOUT}

Figure 1 shows the layout of our thruster consisting of a micromachined array of Silicon capillaries and extractor electrodes, each etched from an SOI wafer, passively aligned, and mounted on a LTCC support providing fluidic and electrical interfaces, as well as integrated heaters and temperature sensors.

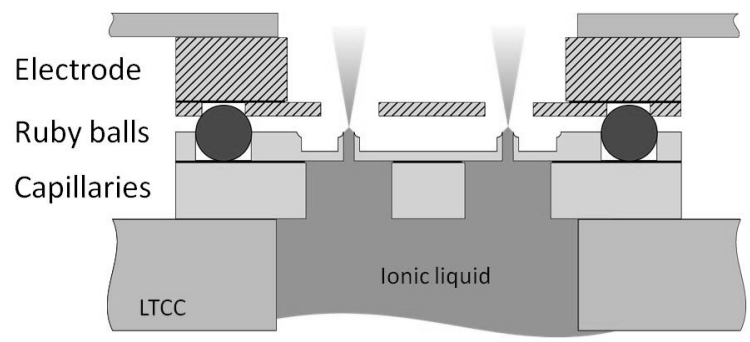

Figure 1: Cross section of the assembled thruster (not to scale). The extractor electrode sits on the capillary chip (emitter) spaced by ruby balls. The capillary chip and a fluidic interface are glued onto a ceramic support.

\section{DEVICE FABRICATION}

We have made two types of devices, one type where the extractor electrodes can be individually addressed (figure 2) and one type where the extractor electrodes are all at the same potential (figure 3). We will focus in this paper on the latter case.

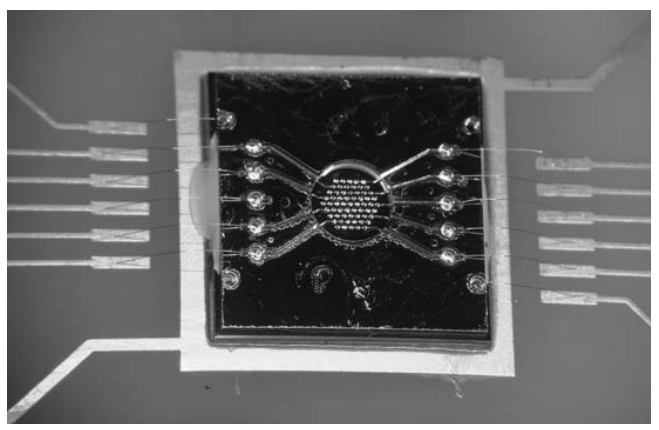

Figure 2: Photograph of a fully assembled thruster in the individually addressable extractor electrodes configuration.

Figure 3 is a SEM image of the assembled capillaries with integrated extractor electrodes providing an individual extractor aperture for each emitter. Extractor and capillary chips are separated by ruby balls serving as insulators and alignment features. This assembly is connected to a pressurized reservoir by means of an 
Upchurch Scientific Nano-port attached to the backside of the LTCC interface. Figure 4 provides a detailed view of the capillary, with a stand-off height of $70 \mu \mathrm{m}$ and an external diameter of $34 \mu \mathrm{m}$ at the tip. The capillary has an inner diameter of $24 \mu \mathrm{m}$ and is filled with $5 \mu \mathrm{m}$ silica microspheres to increase hydraulic impedance, thus avoiding liquid overflow and also enabling operation in ionic regime.

Details of the microfabrication are given in [7]. The process flow for the capillaries is summarized in Figure 5. The shape of the tip of the capillaries is based on a design by Griss et al. [8]. A key step is the use of a silicon nitride layer that serves as a mask during oxidation, preventing the top of the capillaries from being oxidized (Fig 5d-e), thus making a self-aligned oxide mask for the DRIE of the inside of the capillaries.

The extractor electrodes were fabricated from an SOI wafer by double-sided DRIE. The process flow is summarized in Figure 6. Final assembly relied on passive alignment (accurate to $2 \mu \mathrm{m}$ ) using $200 \mu \mathrm{m}$ ruby spheres. The device can be readily disassembled after operation allowing examination of wear and fuel accumulation.

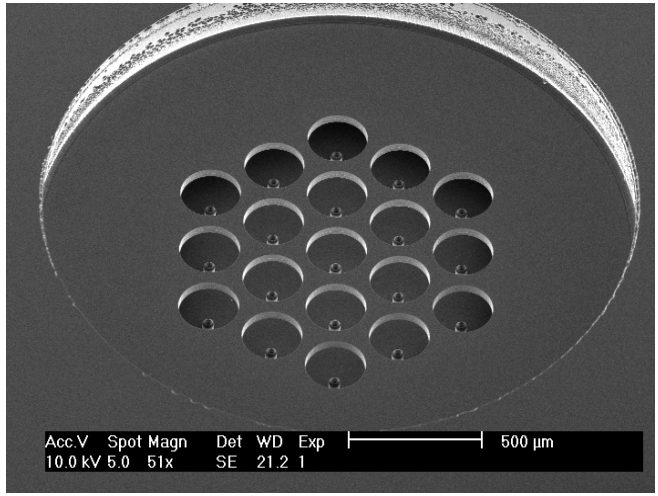

Figure 3: SEM image of an assembled microfabricated colloid thruster consisting of 19 micromachined capillaries and extractor electrodes (extractors are all at the same potential).

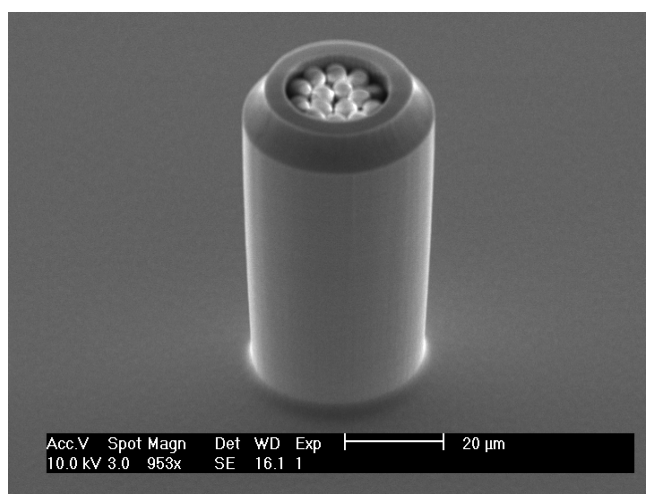

Figure 4: SEM photograph of a single capillary filled with $5 \mu \mathrm{m}$ silica microspheres to increase hydraulic impedance. The standoff height of the capillary is $70 \mu \mathrm{m}$ and the inner diameter is 24 $\mu m$.
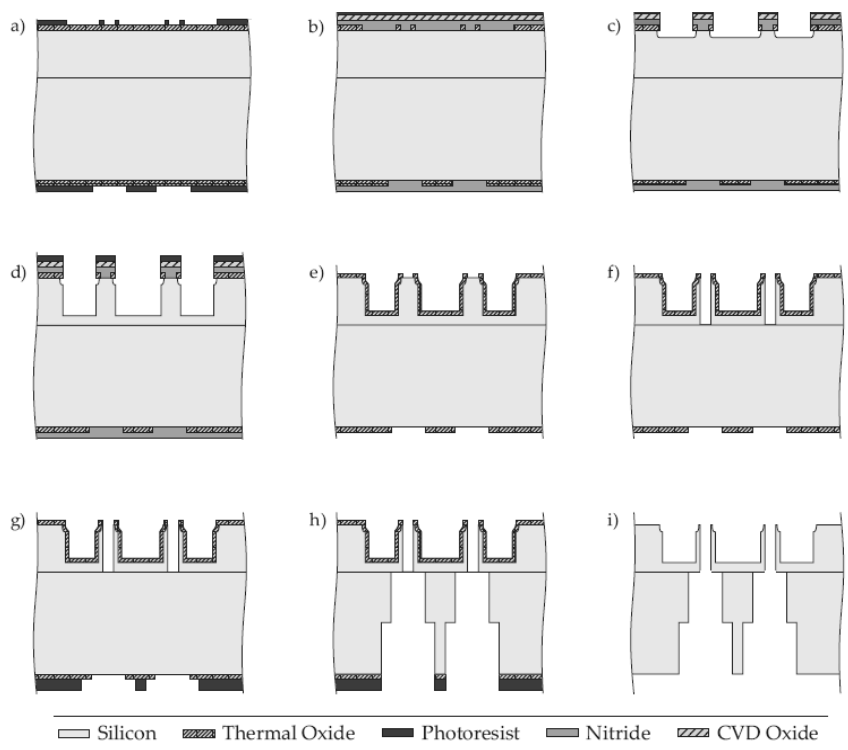

Figure 5: Process flow developed to manufacture large arrays of capillary emitters starting with an SOI wafer. The buried oxide serves as an etch-stop.

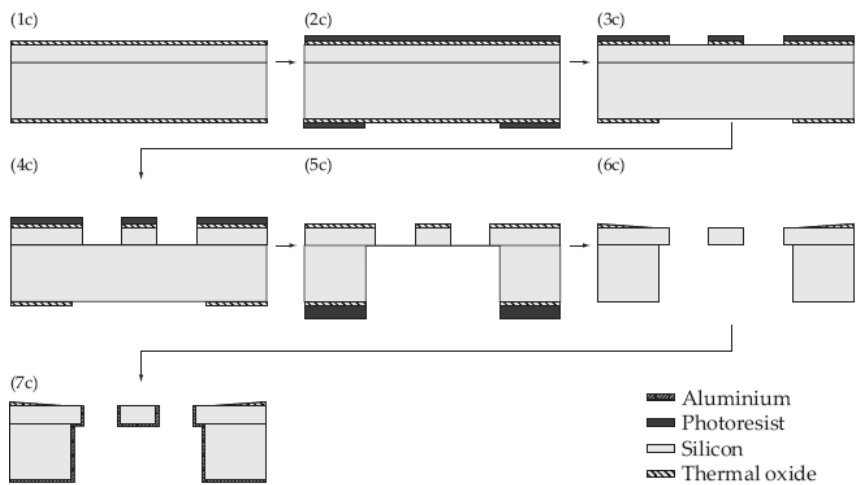

Figure 6: Process flow to fabricate arrays of extraction electrodes from an SOI wafer.

\section{TESTING}

Measuring sub- $\mu \mathrm{N}$ thrust levels is very challenging. As is generally done for the characterization of electric propulsion, the emitted current $I$ was measured, which, when coupled the mass over charge ratio $\mathrm{m} / q$ obtained by mass spectrometry of the emitted particles, and the accelerating voltage $\mathrm{V}$ (assuming beam voltage is equal to the acceleration voltage), allows the thrust $T$ to be determined from:

$$
T=I \sqrt{2 V \frac{m}{q}}
$$

Tests were conducted under ultra high vacuum. The capillary emitters were placed at high voltage while the extractor electrode was grounded. Spray current was measured using a Faraday cup. Tests were carried out successfully with the ionic liquids EMI-BF ${ }_{4}$ and EMI-Tf ${ }_{2} \mathrm{~N}$ (often referred to as EMI-Im). As recommended by Lozano et al. [9] the polarity is switched periodically to 
avoid electrochemical reactions.

Figure 7 shows the measured emitter spray current as a function of time during a voltage ramp for a single capillary emitter filled with $5 \mu \mathrm{m}$ diameter silica beads for EMI-Tf ${ }_{2} \mathrm{~N}$. The current strongly depends on the voltage. There is no electrospray below a critical voltage that depends on device geometry and on the surface tension of the sprayed liquid [10].

In order to determine the energy distribution of the particles in the emitted beam, a retarding voltage $V_{\text {ret }}$ was applied to a grid placed in front of the Faraday cup. Figure 8a shows typical data for an emitter at $900 \mathrm{~V}$ : as $\mathrm{V}_{\text {ret }}$ is increased an increasing portion of the beam is blocked. Differentiating the current vs. $V_{\text {ret }}$ curve yields the density distribution of the various particle energies inside the beam, plotted in figure $8 \mathrm{~b}$. One main peak can be seen around $800 \mathrm{~V}$. These results correspond to measurements by other groups using single non-micromachined capillaries, indicating ionic mode operation. Figure 9 shows the evolution of the density distribution as a function of emitter potential. We interpret the curves as corresponding to pure ionic regime up to $1100 \mathrm{~V}$, while above $1100 \mathrm{~V}$ mixed ion-droplet emission sets in. Further details can be found in [11]. We have observed this behavior on single capillaries as well as on arrays of 19 capillaries with a $250 \mu \mathrm{m}$ pitch (density 1800 capillaries $/ \mathrm{cm}^{2}$ ).

To unambiguously confirm pure ion mode operation, time of flight (TOF) mass spectrometry was done by K. Smith on the beam emitted from an array of 19 capillaries (as in figure 3) spraying the liquid EMI-BF 4 . [11] The charge to mass ratio can be computed from the particle time of flight and acceleration voltage. The TOF data in Figure 10 shows two steps, corresponding to the atomic mass of the monomer $\mathrm{EMI}^{+}$and of the dimer (EMI$\left.\mathrm{BF}_{4}\right) \mathrm{EMI}^{+}$. The beam hence consists almost exclusively of ions.

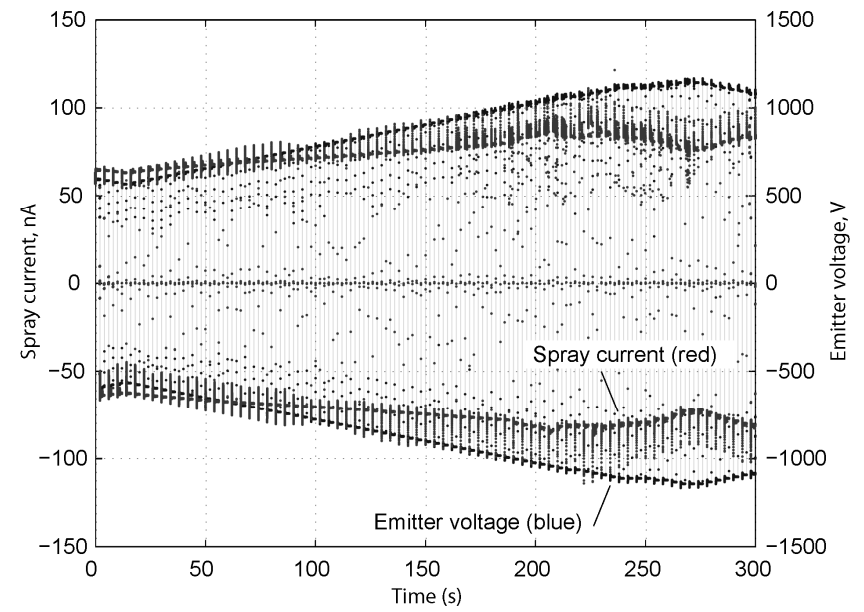

Figure 7: Modulation of spray current by a changing the emitter voltage for a single capillary
From the TOF data the thrust and specific impulse can be computed, as displayed in figure 11 as a function of extraction voltage. The specific impulse, a measure of the change in momentum per unit of propellant, of our nanoelectrospray source is $3500 \mathrm{~s}$ at $1.2 \mathrm{kV}$, corresponding to an ion velocity of $35.10^{3} \mathrm{~m} / \mathrm{s}$. The computed thrust is 20 $\mathrm{pN}$ per emitter, so a total thrust of $100 \mu \mathrm{N}$ would be achievable on a 4 " wafer.

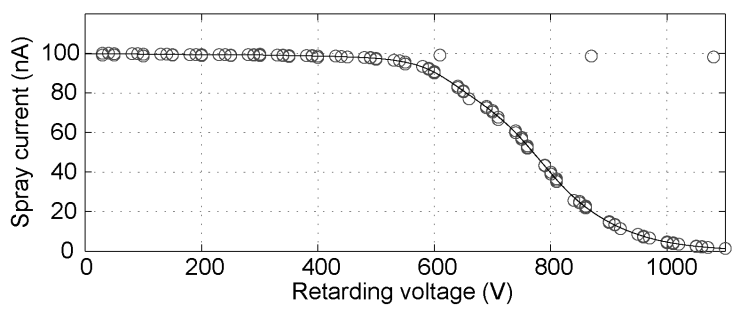

(a)

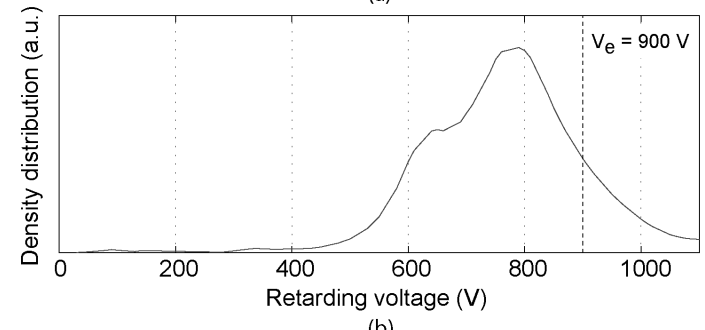

(b)

Figure 8: Retarding potential analysis of the electrospray. The emitter voltage was $V_{e}=900 \mathrm{~V}$.

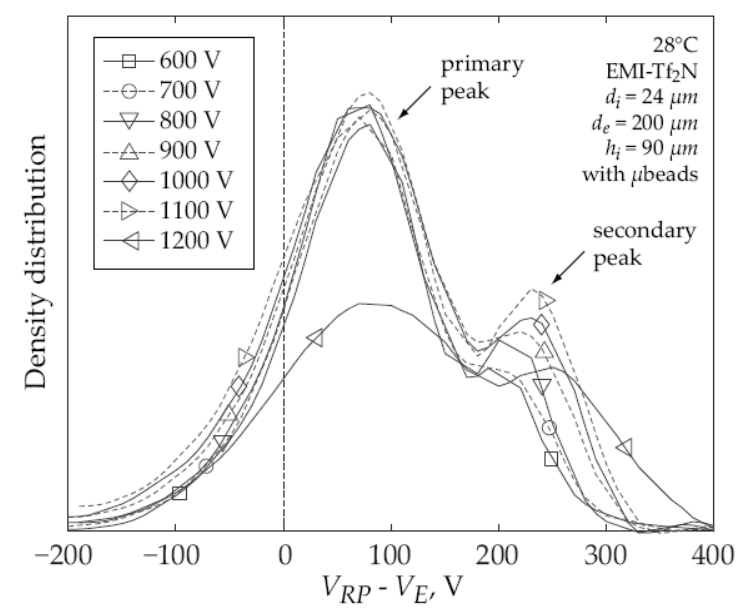

Figure 9: Retarding potential distribution measurements at constant flow rate for a $24 \mu \mathrm{m}$ i.d. capillary with a $200 \mu \mathrm{m}$ extraction electrode spaced at $90 \mu \mathrm{m}$ from the capillary tip and filled with $5 \mu \mathrm{m}$ microbeads spraying EMI-Tf ${ }_{2} N$. The emitter voltage is subtracted from the retarding voltage to show the similarity of energy distributions for different emitter voltages. The change in beam properties for voltages above $1100 \mathrm{~V}$ is clearly visible. 


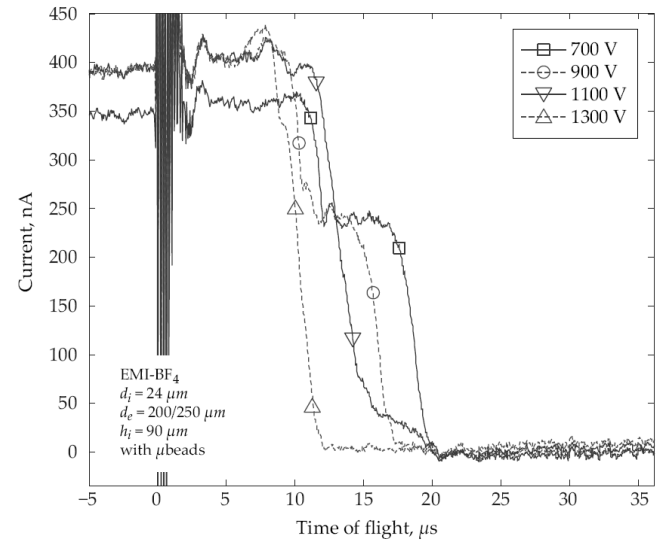

Figure 10: Time-of-flight traces recorded with a 19-emitter array with capillaries having a 24 um inner diameter, filled with $5 \mu \mathrm{m}$ microbeads with an extractor electrode with $200 \mu \mathrm{m}$ holes spaced at $90 \mu \mathrm{m}$ from the capillary tip.

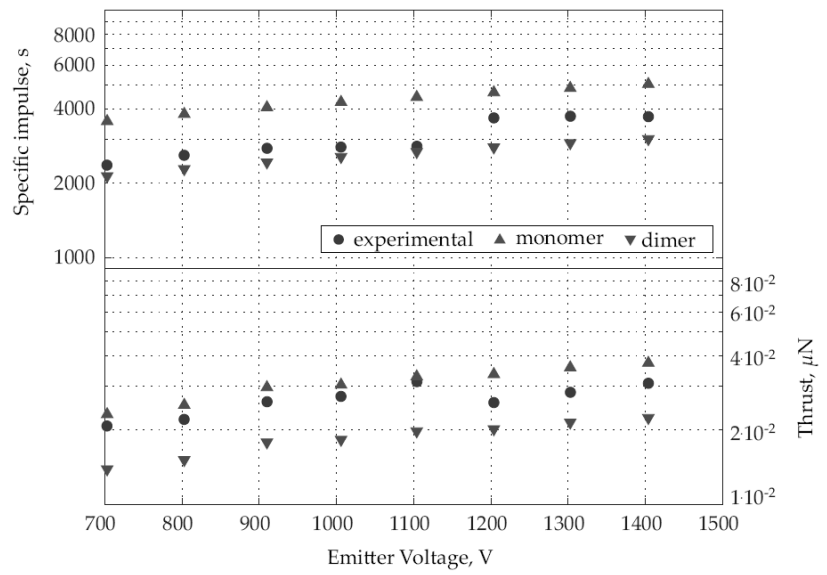

Figure 11: Plot of the specific impulse and thrust determined from the experimental TOF traces recorded for a 19-emitter array. The experimental data is compared to theoretical values for monomers and dimers of the ionic liquid EMI-BF4.

\section{CONCLUSIONS}

We have demonstrated the successful operation of electrospray thrusters consisting of arrays of microfabricated silicon capillaries with integrated extraction electrodes. We have achieved high specific impulse, and shown that the device can work in either pure ion regime or in mixed regime for two ionic liquids. Stable operation was shown for tens of hours. Such a MEMS thruster is a promising candidate for propulsion systems for formation flying missions as well as for pico- and nano-satellites.

\section{ACKNOWLEDGMENTS}

We thank J. Stark and K. Smith of QMUL for helpful discussions and for the TOF data, and ESA for partial financial support.

\section{REFERENCES}

[1] R. Krpoun, M. Räber, H.R. Shea, "Microfabrication and test of an integrated colloid thruster", in Proc. 21st IEEE Int. Conf. on Micro Electro Mechanical Systems (MEMS 2008), pp. 964-967, 2008

[2] J. Fenn, M. Mann, C. Meng, S. Wong, C. Whitehouse, "Electrospray ionization for mass spectrometry of large biomolecules", Science 246 (4926), 64-71, 1989

[3] I. Romero-Sanz, R. Bocanegra, J. Fernandez de la Mora, J. "'Source of heavy molecular ions based on Taylor cones of ionic liquids operating in the pure ion evaporation regime", J. Appl. Phys. 94, 3599-3605, 2003

[4] R. Krpoun, "Micromachined Electrospray Thrusters for Spacecraft Propulsion", PhD thesis, Ecole Polytechnique Fédérale de Lausanne, No. 4255, 2008.

[5] A. Zorzos, and P. Lozano, 'The Use of Ionic Liquid Ion Sources (ILIS) in FIB Applications" in Proc. 52nd Int Conf. on Electron, Ion, and Photon Beam Technology and Nanofabrication (Portland), 2008

[6] P. Lozano, "Energy properties of an EMI-Im ionic liquid ion source", J. Phys. D: Appl. Phys. 39, 126134. 2006

[7] R. Krpoun and H. Shea, "Integrated out-of-plane nanoelectrospray thruster arrays for spacecraft propulsion", Journal of Micromechanics and Microengineering, 19, p. 045019, 2009

[8] P. Griss et al., "Development of micromachined hollow tips for protein analysis based on nanoelectrospray ionization mass spectrometry", Journal of Micromechanics and Microengineering 12, pp. 682687, 2002

[9] P. Lozano and M. Martinez-Sanchez, "Electrospray emission from nonwetting flat dielectric surfaces", $J$. Colloid Interface Sci. 280, pp 149-154, 2004

[10] R. Krpoun and H. Shea, "A method to determine the onset voltage of single and arrays of electrospray emitters", Journal of Applied Physics, 104, p 064511, 2008

[11] R. Krpoun, H. R. Shea, K. L. Smith and J. P. W. Stark, "Tailoring the hydraulic impedance of out-ofplane micromachined electrospray sources with integrated electrodes", to appear in Applied Physics Letters, 2009

\section{CONTACT}

* Herbert Shea, herbert.shea@epfl.ch http://lmts.epfl.ch 